\title{
Hypertrophic cardiomyopathy in young triathlete patient: case report
}

\author{
Camila Alcalde Mazza*, Edmo Atique Gabriel and Marina Alves Jacintho de Mello \\ Union of the Colleges of the Great Lakes- UNILAGO, Brazil
}

\begin{abstract}
Discuss the importance of early diagnosis of hypertrophic cardiomyopathy in young triathlete patient and the main preventive and therapeutic measures. O.P.S. J, male sex, 45 years, sought cardiology attendance, in urgent character, due to palpitations and dyspnea. His life habits consisted of being a triathlete without medical accompaniment, performing exaggerated supplementation with creatine and amino acids, with eventual use of hormones, and daily consumption of 10 to 12 eggs. Asymmetric hypertrophic cardiomyopathy should be diagnosed early, since surgical treatment can be postponed by means of lifestyle changes and use of specific medications.
\end{abstract}

\section{Introduction}

Hypertrophic Cardiomyopathy (HCM) is an autosomal dominant congenital disease linked to a genetic mutation in the heavy betamiosin chain. It is characterized by obstruction of the left ventricular outflow tract with asymmetric septal hypertrophy associated with myofibrillar disarray and interstitial fibrosis, leading to diastolic dysfunction and myocardial ischemia, which may predispose to the appearance of malignant ventricular arrhythmias, leading to syncope or sudden death [1]. Over 400 different mutations were identified with a marked variation in disease penetration and clinical expression [2].

Two-dimensional echocardiography is the basis for imaging, although magnetic resonance imaging (MRI) and computed tomography (CT) provide alternatives [2]. In more complex cases, transthoracic echocardiography and MRI of the heart acquire essential role both for diagnosis and for therapeutic planning [3]. The objective of this report is to discuss the importance of early diagnosis of the disease and the main preventive and therapeutic measures.

\section{Case report}

O.P.S.J, male, 45 years old, sought emergency cardiology due to palpitations and dyspnea. His life habits consisted of being a triathlete without medical supervision, performing exaggerated supplementation with creatine and amino acids, with eventual use of hormones, in addition to daily consumption of 10 to 12 eggs.

MRI of the heart, performed in 2011, at 38 years, revealed asymmetric hypertrophy, associated with significant ventricular obstruction and presence of apical aneurysm (Figures $1 \mathrm{a}$ and $1 \mathrm{~b}$ ).

Regarding the interpretation of the examination, left asymmetric left ventricular myocardial hypertrophy with septal predominance, with a greater wall thickness of $23 \mathrm{~mm}$ in the medial inferosseptal segment, with left ventricular diastolic dysfunction, midventricular obstruction and apical left ventricular aneurysm formation were evidenced. presence of extensive myocardial fibrosis ( 58.6 grams or $26.8 \%$ of the left ventricular mass).
Clinical treatment and lifestyle changes were chosen and, in 2017, new MRI showed absence of ventricular obstruction and maintenance of other findings. In addition, periodic echocardiograms were performed to monitor the condition (Figures $2 \mathrm{a}$ and $2 \mathrm{~b}$ ).

Examination compatible with asymmetric apical hypertrophic cardiomyopathy medio ventricular and aneurysm formation in the left ventricle and the presence of extensive myocardial fibrosis $(22 \%$ left
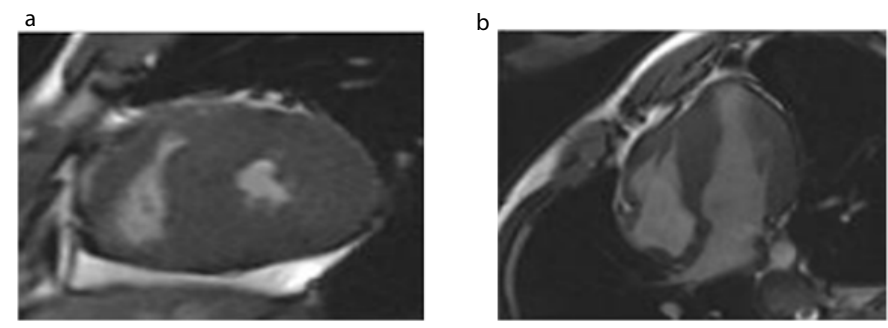

Figure 1. Extensive left ventricular hypertrophy with outlet obstruction
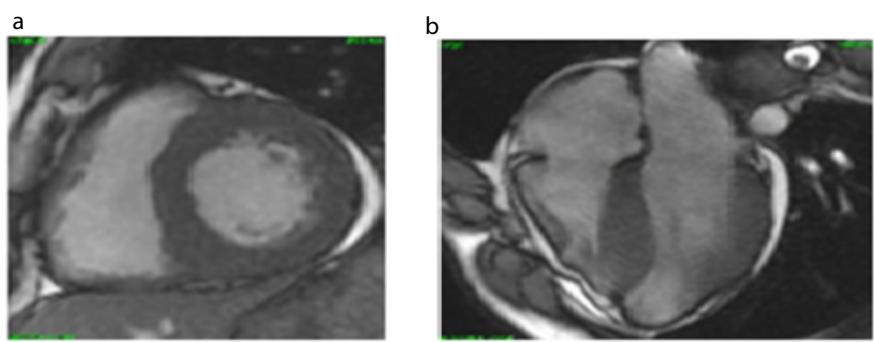

Figures 2. Left ventricular hypertrophy with reduced outlet obstruction

*Correspondence to: Camila Alcalde Mazza, Avenue José Munia, 6300- São José do Rio Preto/SP- Brazil; Tel: 5517997884321; E-mail: mazzacamila5@hotmail.com

Key words: early diagnosis, young triathlete, hypertrophic cardiomyopathy

Received: November 31, 2018; Accepted: December 11, 2018; Published: December 14, 2018 
ventricular mass). Regarding the previous examination (11/16/2011), there is an increase in the apical aneurysm region, with an extension of stable myocardial fibrosis.

The patient presented significant improvement of the symptoms, not needing until the present moment of surgical approach. The clinical treatment adopted is: angiotensin receptor blocker, beta-blocker and potassium-sparing diuretic.

\section{Discussion}

The echocardiogram (ECHO) is the method that confirms the diagnosis and allows evaluation for decision making. Among the parameters to be evaluated are: cavity dimensions; location of hypertrophy; presence of intraventricular gradient, anterior mitral systolic movement and mitral regurgitation; granular aspect of the myocardium; diastolic function [4]. The diagnosis is confirmed by the presence of septum thickness or free wall greater or equal to $15 \mathrm{~mm}$ or greater or equal to $13 \mathrm{~mm}$ in patients with a first-degree family history with the disease, and this hypertrophy is not explained by the presence of other cardiac or systemic diseases [5].

MRI has been established in the approach of HCM [6]. This examination is indispensable for adequate knowledge of the anatomy and stratification of risk of sudden death. It is emphasized that this method not only allows to define with more trustworthiness the form of cardiomyopathy but also identifies the presence of findings difficult to recognize to ECHO and of prognostic importance, such as apical aneurysm and mitral insufficiency. Still, it is the only non-invasive test capable of providing information about myocardial fibrosis, which seems to be the best predictor of sudden death in HCM [7]. In this case of the article, MRI emphasized the presence of apical aneurysm and myocardial fibrosis.

As for treatment, it is reserved for symptomatic patients and can be divided into pharmacological and invasive interventions. Beta- blockers are the main drugs in the pharmacological treatment of $\mathrm{HCM}$, relieve symptoms in $2 / 3$ of patients and reduce obstruction in the left ventricular outflow tract during exercise, being the drug of choice for these patients. Calcium channel blockers are an alternative to beta-adrenergic blockade. In patients who respond inadequately to monotherapy, the association of the two drugs may be attempted [5].

Invasive treatment is indicated only for symptomatic patients, class III and IV (New York Heart Association - NYHA), refractory to optimized medication and who have gradients in the left ventricular outflow tract. Aortic transvalvarcardiomyectomy is the procedure with the longest experience and considered the gold standard [8].

It is concluded that asymmetric hypertrophic cardiomyopathy should be diagnosed early because surgical treatment may be postponed by means of changes in lifestyle and use of specific medications.

\section{References}

1. Brazão de Oliveira, Marcos Aurélio (2002) Hypertrophic cardiomyopathy, physical activity, and sudden death. Rev Bras Med Esporte.

2. Goldman L, Ausiello D (2005) Cecil. Internal Medicine Treaty. Elsevier

3. Casolo GC, Poggesi L, Boddi M, Fazi A, Bartolozzi C, et al. (1987) ECG-gated magnetic resonance imaging in right ventricular dysplasia. Am Heart J 113: 1245-1248. [Crossref]

4. St John Sutton MG, Plappert TJ (1996) Cardiomyopahty. In: St John Sutton MG et al - Textbook of Echocardiography and Doppler in Adults and Children. 2nd ed Massachusetts: Blackwell Science. 375-384.

5. Moreira MCV, Montenegro ST, Paola AAV (2015) Hypertrophic cardiomyopathy. Textbook of the Brazilian Society of Cardiology 2. Edition.

6. Bittencourt MI, Rocha RM, Albanesi filho FM (2010) Hypertrophic cardiomyopathy. Rev Bras Cardiol 23: 17-24.

7. Ilan Gottlieb, Gabriel C Camargo, Maria Eduarda Derenne (2014) Magnetic Resonance in Hypertrophic Cardiomyopathy. JACC: Cardiovas Imag 27: 202-207.

8. Wynne J, Braunwald E (1997) The cardiomyopathies and myocarditides - hypertrophic cardiomyopathy In: Braunwald E (ed) - Heart Disease - A Textbook of Cardiovascular Medicine, 5th ed. Philadelphia: WB Saunders 1414-1426.

Copyright: (C2018 Mazza CA. This is an open-access article distributed under the terms of the Creative Commons Attribution License, which permits unrestricted use, distribution, and reproduction in any medium, provided the original author and source are credited. 